\title{
Application and Development of Carbon Nanotubes in Manufacturing
}

\author{
Jiaji Su* \\ Lehigh University, Bethlehem, PA18015, USA \\ sujiaji926@163.com
}

\begin{abstract}
Decades after the concept of carbon nanotubes was proposed and became a hot topic of research, there is a new focus on the application of such materials with excellent chemical and physical properties in manufacturing. After investigating academic reports on nanoscale manufacturing in the past few years, this paper discusses and summarizes various potential applications of carbon nanotubes, both electrical and non-electrical. Moreover, with the increasing computer computing power and the improvement of machine learning algorithms, study of CNT based on AI becomes a new and effective way to learn more about this material. However, there are still some unknown properties of carbon nanotubes, which may influence the quality control of products in the process of manufacturing, and the harm to human body and the natural environment is also an obstacle to its practical application and manufacturing.
\end{abstract}

\section{CCS CONCEPTS}

- Social and professional topics $\rightarrow$ Professional topics; Computing and business; Economic impact.

\section{KEYWORDS}

Manufacturing, AI, Carbon Nanotube, Computer Chip, Semiconductor

ACM Reference Format:

Jiaji Su. 2021. Application and Development of Carbon Nanotubes in Manufacturing. In 2021 2nd Asia-Pacific Conference on Image Processing, Electronics and Computers (IPEC2021), April 14-16, 2021, Dalian, China. ACM, New York, NY, USA, 4 pages. https://doi.org/10.1145/3452446.3452632

\section{INTRODUCTION}

In the past few decades, nanoscale manufacturing has developed rapidly, mainly in the form of one to 100 nanoscale components in a single manufacturing process. At present, nano manufacturing is mainly used in computing [1], electronics and electrical [2], medical care [3], photonics, biotechnology, etc. Carbon nanostructure is the most commonly used structure in nanotechnology. It

\footnotetext{
${ }^{*}$ Corrreponding author

Permission to make digital or hard copies of all or part of this work for personal or classroom use is granted without fee provided that copies are not made or distributed for profit or commercial advantage and that copies bear this notice and the full citation on the first page. Copyrights for components of this work owned by others than ACM must be honored. Abstracting with credit is permitted. To copy otherwise, or republish, to post on servers or to redistribute to lists, requires prior specific permission and/or a fee. Request permissions from permissions@acm.org.

IPEC2021, April 14-16, 2021, Dalian, China

(C) 2021 Association for Computing Machinery.

ACM ISBN 978-1-4503-8981-5/21/04 ..\$15.00

https://doi.org/10.1145/3452446.3452632
}

has the following types: amorphous carbon, graphite/graphene, carbon nano-diamond, carbon nanotube and buckyball structure. Among these, carbon nanotubes are the most attractive. By analyzing its geometry and orientation, scientists have found many advantages, including its high thermal and electrical conductivity, good mechanical properties, and no electromigration degradation. Due to the stringent requirements of the preparation method, there is no way to obtain a large number of uniform standards of carbon nanotubes. However, with the development of computer science and the emergence of artificial intelligence, scientists can use AI approach to control and detect the properties of carbon nanotubes more accurately, which also provides more possibility for nanoscale manufacturing.

\section{RESEARCH METHOD OF CNTS BASED ON AI}

Although scientists and manufacturers are full of expectations for the excellent physical and chemical properties of CNT, however, the study of the properties of the new nanomaterials has always been a difficult problem for scientists, traditional laboratory research has more limitations in the face of this nanoscale material, such as accuracy problems and cost problems, but with the increasing computer computing power and the updating and improvement of machine learning algorithms, which makes it possible to develop an integrated artificial intelligence simulation technology to simulate the material properties of nanoscale materials such as carbon nanotubes.

Huang and his team reported an artificial intelligence (AI) simulation method based on molecular dynamics (MD) was to study the heat transport of carbon nanotubes, which can provide strong theoretical support for the future manufacturing of CNT in the field of electronics industry. According to the paper, they use MD simulation to analyze the effect of defects caused by vacancy and the thermal conductivity firstly. After that, fed the data that came out for MD simulation into the artificial intelligence cluster, which is set up to build the explicit relationship between thermal transport of carbon nanotube and system size, chirality and vacancy defect concentration [4].

The AI model can successfully simulate the thermal conductivity situation of carbon nanotubes, through which, the analytical solution that from traditional MD simulation could be supplement. This integration method combines the advantages of high precision, low cost and explicit modeling of artificial intelligence technology. The method required training data based on MD simulations came from specific geometry and temperature. With input data, artificial intelligence technology can generate meaningful solutions of complex problems. The important conclusion that length has the greatest influence on the thermal conductivity of carbon nanotubes. It has a 


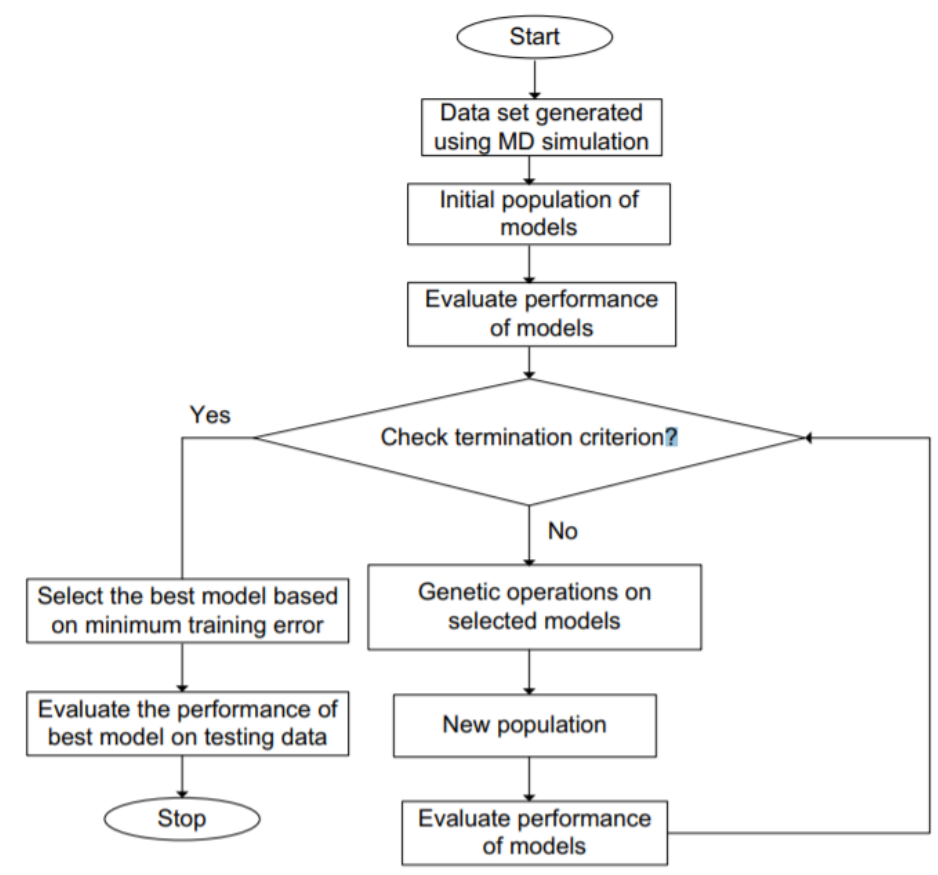

Figure 1: The heat conduction mechanism of nanomaterials is simulated by AI based on MD

theoretical guiding role for CNT in electronic manufacturing, such as chip and diode production (in Figure 1).

\section{ELECTRIC APPLICATIONS OF CNTS}

In the computer chip industry, silicon has always been the undisputed leading role. However, with the continuous improvement of academic research and manufacturing requirements on computer computing power, silicon chips have gradually fallen into the bottleneck of development. In this case, CNTs could be seen as a lifeline for the electronics industry. It is better than silicon in all its physical properties, and smaller in size, so CNTs would be the best successor to silicon-based chips, regardless of cost. If CNTs is successfully applied to the electronic manufacturing industry, it will also greatly improve and enhance the quality of human life.

The process of making chips from carbon nanotubes is complex. This process was discovered and summarized by researchers at the University of Wisconsin-Madison. To put it simply, the researchers first had to pick out the best carbon nanotubes, then combine them on a substrate in a specific sequence and heat them in a vacuum. However, from a research point of view, there are still many hurdles to overcome. This is completely different from traditional silicon chip manufacturing, so the production process also need to be redesigned.

According to the size and shape of carbon nanotubes, the properties of carbon nanotubes can be divided into metal and semiconductor types. The biggest problem with using carbon nanotubes as transistors is that they are a mixture of the two properties. When the two types of transistors are glued to each other in a linear or beam fashion, they lose most of their function. Because only

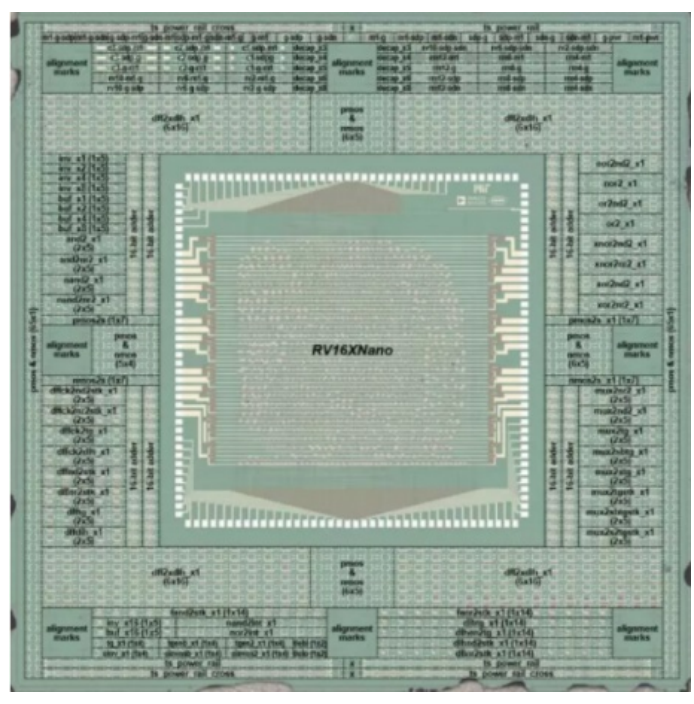

Figure 2: Microscopic image of RV16XNANO

semiconductor-based nanotubes can be made into transistors, and when the two types of transistors stick together, the metal properties are more powerful than the semiconductor properties. It is not only tedious but also inefficient to process the nanotubes one by one. So, the biggest hurdle for carbon nanotubes as transistors is that there is no easy way to separate the metal from the semiconductor. 
In August 2019, MIT Professor, Gage Hills et al. [5] reported in The journal, Nature, a major advance in the field of carbon nanotube chip manufacturing. They developed and produced a 16-bit microprocessor made entirely from carbon nano-transistors. It is the largest computer chip ever made of carbon nanotubes. They named the processor RV16X-Nano, a 16-bit microprocessor based on the RISC-V instruction set that runs standard 32-bit instructions on 16-bit data and addresses, contains more than $14,000 \mathrm{cmos} \mathrm{CN}-$ FEts, and is designed and manufactured using industry-standard processes. The breakthrough proves that it is no longer a fantasy to build microprocessors that surpass silicon entirely from carbon nanotube field effect transistors (CNFETS).Specifically, Hills and colleagues propose a fabrication method that combines treatment and design techniques to overcome nanoscale defects across the entire wafer at the macro scale. They used a peeling process to prevent the nanotubes from coalescing in case the transistors failed to work properly. In addition, the circuit has been carefully redesigned (in Figure 2).

In the field of electronic manufacturing, CNTs does have excellent performance, so it has attracted many researchers to further study this field. However, most of them are still in the experimental stage, and it is still difficult to design and manufacture them in accordance with industry standards.

\section{NONELECTRIC APPLICATIONS OF CNTS}

In the non-electrical field, there have also been many studies using CNTS in combination with other metal elements. Examples include gallium and CNT. Because gallium has the largest liquid metal temperature range and lower vapor pressure at high temperatures, Gao et al. [6] reported that their research has successfully combined carbon nanotubes with gallium to make nanoscale thermometers. The results show that the nanometer thermometer can measure the temperature range of 50-500, which is sufficient for the temperature testing range required by most experiments. CNTS thermometers also outperformed traditional mercury thermometers in accuracy. However, using the thermometer requires the aid of a scanning electron microscope, and the product may be difficult to get out of the laboratory and into people's daily lives.

Another use of CNTs is membrane distillation. The main method to filter $\mathrm{NaCl}$ from seawater is reverse osmosis, which is commonly used to produce drinking water. This principle is also used in CNT membrane distillation. The process USES a polymer film such as polytetrafluoroethylene and polyester. Other types of membrane distillation use similar membrane materials, but less popular. In some cases, hydrophobic coatings are applied to membranes to increase the repulsion of solutes and increase the speed of water molecules. Carbon nanotubes show flux properties that can exist in existing membranes. Some academic studies have shown that CNTs can be used to prepare reverse osmosis membranes or grow new membranes based on CNTs. Moreover, CNTS have been added to existing membranes to improve performance. There are many different ways to do this to achieve a certain purity and desired edge structure, but the end result is a polymer membrane with dispersed CNTs. The result shows that carbon nanotubes can increase resistance to membrane fouling.
Zhang and his team produced a new type of material called Carbon Nano-paper based on CNTS in the laboratory [7] [7], which consists of overlapping networks of carbon nanotubes or singlewalled/multi-walled carbon nanotubes. Based on the excellent mechanical, electrical, and thermal properties of carbon nanotubes, these porous materials are flexible, expandable, and thermal conductive, and are primarily used for heat dissipation, wave absorption, and structural enhancement. This new material based on CNTS has many application prospects. For example, based on its excellent thermal conductivity and flexibility, carbon nano-paper could be designed to be attached to a chip, which would make an ideal cooling device. During the preparation, Zhang and his team overcame the Van Der Waals forces due to the CNTS between the tubes.

Research shows that CNTs also have many potential applications in non-electrical fields. Many difficulties may be encountered when CNTS are directly used for manufacturing, but many benefits will be achieved if CNTS are used as an auxiliary material to improve performance. The fabrication of new materials by changing the intermolecular arrangement of CNTS is also an interesting direction.

\section{DISCUSSION AND CONCLUSION}

Although carbon nanotubes have been studied for more than 40 years, there are still some problems in producing them on a large scale. The first is that carbon nanotubes cannot be produced in exactly the same size, and they cannot be stacked up for use if they are not in the same length. The second problem is that the quality of the nanotubes cannot be controlled when they are put into mass production. Carbon nanotubes are rolled up from graphene, so the direction and radius of the coil affect the properties of the nanotubes. Without a unified process, the chemical and physical properties of the product cannot be controlled. Third, because of the price, they cannot be used in large quantities in industrial production unless scientists find better ways to produce them in the future.

Although CNTS have shown outstanding advantages in use and enhanced engineering performance, the current health benefits of CNTS are also the main reason for their inability to enter the manufacturing sector, and the degradation of CNTS is extremely low. If they are introduced into the environment, they will stay there for a long time. In the current brief study, the impact on health and the environment has not been fully understood. Studies have shown that carbon nanotubes are difficult to degrade and that their length determines the toxin. This limited her use in manufacturing. Because of insufficient research, it is also difficult to determine whether CNTS can be filtered from the distillation membrane. These safety issues also call for further research and the establishment of safety guidelines for carbon nanotubes in their future use.

\section{REFERENCES}

[1] X. Zhang, C. Sun, and N. Fang, "Manufacturing at Nanoscale: Top-Down, Bottomup and System Engineering," J. Nanoparticle Res., vol. 6, no. 1, pp. 125-130, Feb. 2004, doi: 10.1023/B:NANO.0000023232.03654.40.

[2] M. De Volder and S. Tawfik, "Carbon Nanotubes: Present and Future Commercial Applications." Science Magazine, 2013.

[3] P. Avouris and Z. Chen, "Carbon-based electronics."

[4] Y. van de Burgt, "Laser-assisted growth of carbon nanotubes-A review," J. Laser Appl., vol. 26, no. 3, p. 032001, Aug. 2014, doi: 10.2351/1.4869257.

[5] G. J. Brady, A. J. Way, N. S. Safron, H. T. Evensen, P. Gopalan, and M. S. Arnold, "Quasi-ballistic carbon nanotube array transistors with current density exceeding 
Si and GaAs," Sci. Adv., vol. 2, no. 9, p. e1601240, Sep. 2016, doi: 10.1126/sciadv. 1601240

[6] G. Hills et al., "Modern microprocessor built from complementary carbon nanotube transistors," Nature, vol. 572, no. 7771, pp. 595-602, Aug. 2019, doi: 10.1038/s41586019-1493-8.
[7] D. Franklin et al., "Sub-10 nm Carbon Nanotube Transistor," Nano Lett., vol. 12, no. 2, pp. 758-762, Feb. 2012, doi: 10.1021/nl203701g 